\title{
Características fisicoquímicas de la zona baja de cuatro afluentes urbanos del río Atrato, como una aproximación al análisis de sus aportes contaminantes
}

\section{Physicochemical characteristics of the lower area of four tributaries of the Atrato River, as an approximation to the analysis of its polluting contributions}

\section{Zoraida Quesada Martínez*}

\section{Resumen}

Se realizó una evaluación de la calidad fisicoquímica del agua en las quebradas El Caraño, La Yesca, y los ríos Quito y Cabí. En cada uno se establecieron 3 puntos de muestreo en la zona de desembocadura al río Atrato. Se monitorearon variables in situ y se tomaron muestras para análisis en laboratorio. El análisis de varianza determinó como variables estadísticamente significativas $(p<0,05)$ al oxígeno disuelto, TDS, sólidos suspendidos, turbidez, conductividady nitratos, las cuales presentaron influencia sobre la problemática de contaminación. El análisis de correspondencia sin tendencia (DCA) agrupó las variables: sólidos suspendidos, turbidez y nitratos como las variables de importancia en la calidad del recurso hídrico asociadas con contaminación por minería en el río Quito y Cabí y a contaminación por vertimientos domésticos en las quebradas El Caraño y La Yesca.

Palabras clave: Actividad minera, Contaminación, Quebradas El Caraño y La Yesca, Residuos domésticos, Ríos Quito y Cabí, Variables fisicoquímicas.

\section{Abstract}

An evaluation of the physicochemical quality of the water was made in the Caraño, La Yesca, and Quito and Cabístreams. In each sampling station, 3 sampling points were established in the mouth area with the Atrato River. in situ variables were monitored and samples taken for water laboratory analysis. Two samples were taken in 2016. The Kruskal-Wallis analysis determined as statistically significant variables $(p<0.05)$ dissolved oxygen, TDS, suspended solids, turbidity, conductivity and nitrates, which had an influence on the problem of contamination. The
Ingeniera Ambiental y Sanitaria, Especialista en Gestión Integral de Cuencas Hidrográficas, Investigadora Asociada tipo II, Instituto de Investigaciones Ambientales del Pacífico (IIAP), Quibdó, Colombia.

e-mail: zquesada@iiap.org.co 


\section{Características fisicoquímicas de afluentes del río Atrato. z Quesada Martínez}

trend less correspondence analysis (DCA) grouped the suspended solid variables, turbidity and nitrates as the variables of importance in the quality of the water resource associated with pollution by mining in the Quito and Cabi rivers and contamination by domestic discharges in the Caraño streams and The Tinder.

Keywords: Domestic waste, Mining activity, Physicochemical variables, Pollution, Quito and Cabi River, El Caraño and La Yesca streams.

\section{Introducción}

Son varios los problemas derivados de la contaminación de los cuerpos de agua; reducción del suministro de agua dulce (Hinrichsen et al. 1998), riesgos en la salud, la inutilización del uso del agua para diversos usos, el impacto negativo sobre la vida acuática (e industria pesquera) y la desaparición del valor estético, son solo algunos de los efectos asociados con calidad de agua (Wall 1981). Desde una perspectiva de manejo de cuencas, y considerando que este constituye un sistema abierto que interrelaciona aspectos biofísicos y socioeconómicos, es posible enunciar que en esta problemática existe una complejidad de soluciones que se deben accionar integralmente de un modo lógico en la variabilidad temporal, espacial e institucional (Faustino 1986).

En su recorrido, el río A trato recoge innumerables afluentes, específicamente en su zona media a la altura del municipio de Quibdó, recibe las aguas de los ríos Quito y Cabí, y las quebradas La Yesca y El Caraño, cada una de estas contaminadas por el desarrollo de diferentes prácticas domésticas y socioeconómicas, generando perturbaciones en la calidad fisicoquímica del agua de esta fuente hídrica. A lo anterior se suma un deficiente sistema de saneamiento básico en el municipio lo que trae como consecuencia el vertimiento de aguas residuales domésticas sin tratamiento previo, así como una mala disposición de los residuos sólidos y líquidos derivados de las actividades diarias.

El río Cabí está situado al suroriente del municipio de Quibdó, como una subcuenca de la gran hoya hidrográfica del río Atrato localizada en los municipios de Quibdó y Atrato en el departamento del Chocó. Cuenta con 16.219 hectáreas, equivalente a $162.19 \mathrm{~km}^{2}$ en las cuales se encuentran establecidas las comunidades de Pacurita y Guadalupe, ubicadas en los márgenes del río Cabí, San Martín y San José de Purré en el lecho del río Purré afluente del primero (CODECHOCO 2009). De igual manera, la microcuenca El Caraño, nace en el alto del coronillo al nororiente del municipio de Quibdó, a una altura de 100 msnm, en una zona de bosque muy húmedo tropical (Bmh- $\mathrm{t}$ ), desciende hasta el barrio Kennedy sector las cantoneras a una altura de $43 \mathrm{msnm}$ en donde vierte sus aguas al río Atrato; constituye un sistema lótico de gran importancia para el desarrollo socioeconómico de la parte nororiental del municipio de Quibdó (IIAP 2012). La quebrada La Yesca nace en las inmediaciones del barrio El Jardín sector Suba, al oriente del aeropuerto El Caraño en el punto denominado Alto de Granadillo en la margen izquierda y se extiende por la ciudad de Quibdó, sobre todo por los barrios El Jardín (Castillo, Zona Minera, Los Ángeles), La Esmeralda, Margarita, Yesca Grande, la Yesquita y San Vicente, desembocando al río Atrato. Está ubicada entre 24 y $45 \mathrm{msnm}$. Posee una extensión de 2,42 km; su área de drenaje total llega a los $2,8 \mathrm{~km}^{2}$ (Rojas et al. 2007).

Al igual que estas fuentes urbanas, el río Quito reviste de importancia en el área urbana del municipio de Quibdó; pese a que sus aguas no circundan esta población, constituye una de las principales fuentes hídricas que desembocan en la cuenca media del río Atrato. Se establece en la corriente principal que surca el territorio del municipio de Río Quito, el cual tiene un caudal en verano de $200 \mathrm{~m}^{3} / \mathrm{seg}$ lo que lo hace navegable todo el año. Este río y la mayoría de sus afluentes 


\section{Bioetnia Volumen 13, 2016}

nacen en elCerro de Chachajo en las estribaciones de la Serranía del Baudó. Durante su recorrido recibe las aguas de numerosas fuentes hídricas en las que se destacan las quebradas Grande, Antadocito, Madrevieja, Caripató, Queguedó, y Chigorodó. Otras quebradas menores se presentan como afluentes del río Quito: Paimadocito, Mejardó, Paimadó, Guayacán, El Cano. De igual manera recibe aguas de otras corrientes importantes sobre la margen derecha como lo es el caso del río Paimadó que confluye desde el municipio de Atrato y se une a este al frente de la cabecera municipal (IIAP 2014).

Los principales focos de contaminación de estas fuentes hídricas están determinadas por actividades industriales representadas en grandes aportes hechos por la actividad minera. Por otro lado, en los centros poblados pequeños, en donde los procesos de industrialización son mínimos y hasta escasos, las cuencas y subcuencas hidrográficas se ven influidas por los aportes contaminantes provenientes de actividades domésticas, falta de una infraestructura adecuada para el manejo de residuos sólidos y saneamiento hídrico, además de los aportes provenientes de las actividades productivas. El municipio de Quibdó no es ajeno a esta situación, debido a la inexistencia de un sistema de acueducto con cobertura en el área urbana, que de acuerdo con EPQ (2017) está representada en $30,3 \%$ a diciembre de 2008 . Además, no hay un buen manejo y disposición final de residuos sólidos, evidenciándose focos de contaminación por disposición inadecuada de los mismo y vertimientos directos a las fuentes hídricas provenientes de las viviendas ubicadas en la gran mayoría de los casos, en el área de cauce de las subcuencas; el alcantarillado sólo cubre un porcentaje de la capital (17\% a diciembre de 2008) y el resto tiene soluciones individuales como pozos sépticos que afectan las características fisicoquímicas y ecológicas de las subcuencas de los ríos Cabí, las quebradas El Caraño y La Yesca, las cueles son afluentes de la cuenca hidrográfica del río Atrato. A esta situación se suma la práctica de una minería tecnificada ilegal que contribuye con grandes volúmenes de vertimientos líquidos con altas cargas contaminantes a las fuentes hídricas principalmente a los ríos Cabí y Quito, otros grandes afluentes del río Atrato.

Todo lo anterior constituye un aporte de contaminantes a la cuenca del río Atrato proveniente del área urbana del municipio, lo cual a su vez puede representar una amenaza a la calidad ecológica de este ecosistema hídrico donde se desarrollan actividades productivas y goza de una importante oferta de bienes y servicios ambientales socioeconómicos y culturales del cual se benefician las comunidades tanto del área urbana, como los centros poblados aguas abajo de la cuenca. Es por ello por lo que el levantamiento y análisis de información sobre las condiciones de calidad fisicoquímica de las fuentes hídricas urbanas de Quibdó, así como la desembocadura del río Quito, permitió tener una aproximación sobre los contaminantes urbanos que ingresan a la cuenca principal del río Atrato a través de la desembocadura de los ríos Quito y Cabí, y las quebradas La Yesca y El Caraño.

Area de muestreo. El área de estudio correspondió a 12 puntos de muestreos ubicados en la zona baja de 4 fuentes hídricas que vierten sus aguas al río Atrato, específicamente en la zona urbana del municipio de Quibdó, para lo cual se establecieron 4 estaciones así: Estación 1 ubicada en la quebrada El Caraño, Estación 2 en el río Quito, Estación 3 en el río Cabí y la Estación 4 en quebrada La Yesca (Tabla 1, Figuras 1 y 2) donde se realizaron muestreos in situ y toma de muestras para análisis en laboratorio de agua. El trabajo de campo fue entre septiembre y noviembre del año 2016.

\section{Método}

Evaluación fisicoquímica del agua. El monitoreo de calidad fisicoquímica de agua y la toma 


\section{Características fisicoquímicas de afluentes del río Atrato. z Quesada Martínez}

Tabla 1. Distribución de puntos de muestreo

\begin{tabular}{|c|c|c|c|c|}
\hline Estación & Fuente hídrica & & Puntos de muestreo & Georeferenciación \\
\hline \multirow[t]{3}{*}{1} & Quebrada El Caraño & 1 & Sector San José & N: $05^{\circ} 41^{\prime} 58.1^{\prime \prime} \mathrm{W}: 7^{\circ} 39^{\prime} 33^{\prime \prime}$ \\
\hline & & 2 & Kennedy sector cantonera & $\mathrm{N}: 05^{\circ} 42^{\prime} 00.09^{\prime \prime} \mathrm{W}: 76^{\circ} 39^{\prime} 38.5^{\prime \prime}$ \\
\hline & & 3 & Desembocadura al río Atrato & N: $05^{\circ} 41^{\prime} 57.6^{\prime \prime} \mathrm{W}: 76^{\circ} 31^{\prime} 44.3^{\prime \prime}$ \\
\hline \multirow[t]{3}{*}{2} & Río Quito & 4 & Corregimiento de Guayabalito & $\mathrm{N}: 05^{\circ} 40^{\prime} 31.5^{\prime \prime} \mathrm{W}: 076^{\circ} 41^{\prime} 05.1^{\prime \prime}$ \\
\hline & & 5 & Corregimiento La Soledad & $\mathrm{N}: 05^{\circ} 40^{\prime} 58^{\prime \prime} \mathrm{W}: 076^{\circ} 40^{\prime} 27.9^{\prime \prime}$ \\
\hline & & 6 & Desembocadura al río Atrato & $\mathrm{N}: 05^{\circ} 41^{\prime} 01.4^{\prime \prime} \mathrm{W}: 076^{\circ} 40^{\prime} 03.1^{\prime \prime}$ \\
\hline \multirow[t]{3}{*}{3} & Río Cabí & 7 & Sector Playita & $\mathrm{N}: 05^{\circ} 40^{\prime} 43.3^{\prime \prime} \mathrm{W}: 76^{\circ} 39^{\prime} 22.9^{\prime \prime}$ \\
\hline & & 8 & Niño Jesús sector Cabí & $\mathrm{N}: 05^{\circ} 40^{\prime} 27.4^{\prime \prime} \mathrm{W}: 76^{\circ} 39^{\prime} 29.4^{\prime \prime}$ \\
\hline & & 9 & Desembocadura al río Atrato & $\mathrm{N}: 05^{\circ} 40^{\prime} 28^{\prime \prime} \mathrm{W}: 76^{\circ} 39^{\prime} 44.8^{\prime \prime}$ \\
\hline \multirow[t]{3}{*}{4} & Quebrada La Yesca & 10 & Barrio San Vicente & $\mathrm{N}: 0.5^{\circ} 41^{\prime} 1,6^{\prime \prime} \mathrm{W}: 76^{\circ} 39^{\prime} 38^{\prime \prime}$ \\
\hline & & 11 & Barrio Niño Jesús & $\mathrm{N}: 0.5^{\circ} 40^{\prime} 58,9^{\prime \prime} \mathrm{W}: 76^{\circ} 39^{\prime} 38,6^{\prime \prime}$ \\
\hline & & 12 & Desembocadura al río Atrato & $\mathrm{N}: 0.5^{\circ} 40^{\prime} 55,3^{\prime \prime} \mathrm{W}: 76^{\circ} 39^{\prime} 44,5^{\prime \prime}$ \\
\hline
\end{tabular}

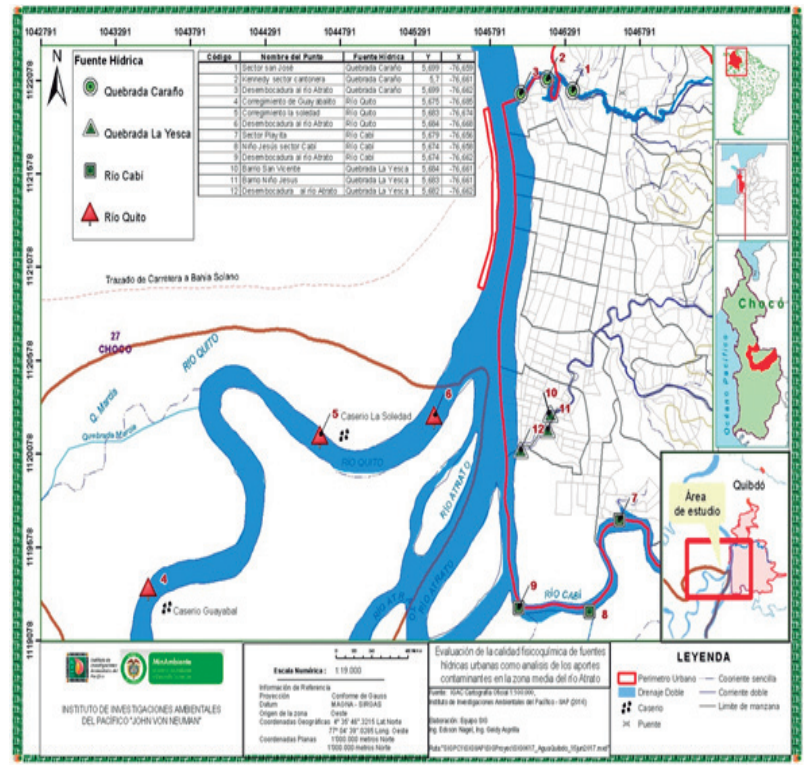

Figura 1. Distribución de puntos de muestreo. Estaciones quebradas La Yesca, El Caraño, ríos Quito y Cabí.

de muestras para análisis en laboratorio se realizó en dos épocas climáticas, correspondiente a tiempo de lluvia y tiempo seco. En cada estación, se tomaron muestras compuestas, siguiendo la metodología establecida por el IDEAM (2013). Se hicieron monitoreo in situ de variables fisicoquímicas como temperatura, conductividad, sólidos disueltos totales, sólidos suspendidos, turbiedad, oxígeno disuelto, $\mathrm{pH}$, nitrato, nitrito y fosfato, utilizando un colorímetro portátil $\mathrm{HACH}$ 850 y un multiparámetros DR 900 . Se tomaron muestras para análisis en laboratorio de aguas de CODECHOCO de parámetros como DBO5, grasas y aceites, siguiendo el protocolo para la toma de muestras establecidos por el laboratorio.

Evaluación del estado y la conservación del agua. Una vez realizado el análisis de la caracterización fisicoquímica se comparó esta información con los estándares de calidad para la preservación de fauna y flora acuática, así como con los datos arrojados por otras investigaciones de calidad de agua en fuentes superficiales urbanas, y las observaciones realizadas en campo sobre el estado del agua y su dinámica de flujo. El análisis de los resultados estará soportado además por un análisis estadístico descriptivo mediante una prueba de Kruskal-Wallis, y de un análisis de correspondencia sin tendencia, a partir de la elaboración de una base de datos construida en una hoja electrónica de cálculo.

\section{Resultados}

Análisis y discusión. Para el caso del oxígeno disuelto $(\mathrm{p}<0,05)$, los datos muestran una disminución en las concentraciones de esta variable 


\section{Bioetnia Volumen 13, 2016}

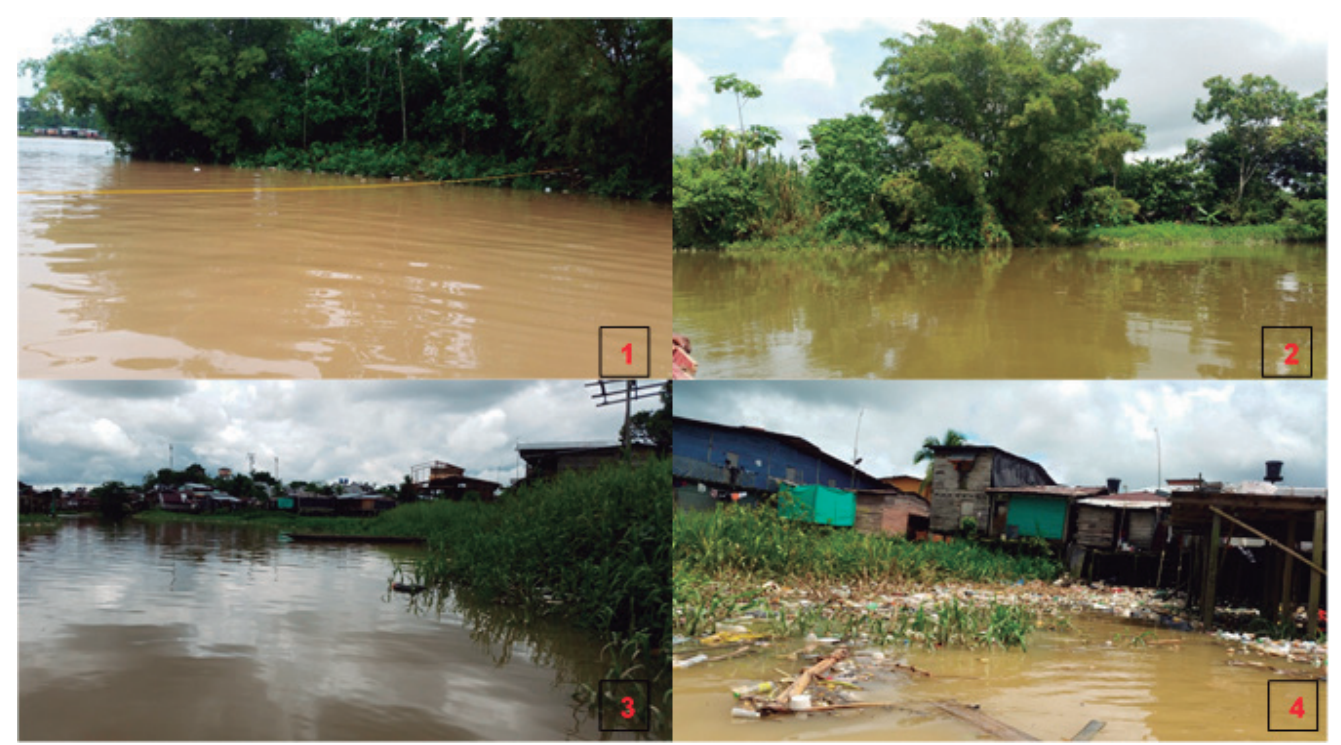

Figura 2. Estaciones de muestreos. 1. Quebrada El Caraño. 2. Río Quito.

3. Río Cabí. 4. Quebrada La Yesca.

en la quebrada La Yesca, estando por debajo del límite establecido por el Decreto 1594/84 del Ministerio de Agricultura para la conservación de organismos acuáticos. En relación con los puntos de muestreo, no existe una diferencia estadísticamente significativa $(\mathrm{p}>0,05)$. Para el caso de la quebrada El Caraño las concentraciones de oxígeno disuelto se presentaron en 5,5 mg/l y $4,72 \mathrm{mg} / 1$ en el punto 3 correspondiente a la desembocadura sobre el río Atrato, permaneciendo dentro del límite admisible para la conservación de la biota acuática (Decreto 1594/84) resultados que contrastan con los presentados en los puntos 1 y 2. La quebrada La Yesca presentó niveles de oxígeno disuelto bajos con un incremento mínimo en su desembocadura con concentraciones de 4,1 $\mathrm{mg} / \mathrm{l}$ y $4 \mathrm{mg} / \mathrm{l}$ en el primer y segundo muestreo respectivamente. La estación 2 (río Quito) y la estación 3 (río Cabí), presentaron niveles óptimos con respecto a esta variable. Los bajos niveles de oxígeno disuelto pudieron estar relacionados con la presencia de microorganismos patógenos que requieren de esta variable en procesos de biodegradación de la materia orgánica, situación que se puede atribuir a la filtración de bacterias provenientes de descargas de aguas residuales directamente sobre estas quebradas sin tratamiento previo, lo que claramente genera limitaciones para el desarrollo de una biota diversa y estable en estos ambientes.

Los niveles de $\mathrm{pH}$ presentaron diferencias estadísticamente significativas entre estaciones de muestreo $(p<0,05)$, con valores que tienden a la acidez en las estaciones de los ríos Quito y Cabí, situación que está directamente relacionada con las actividades mineras que se realizan en estas fuentes hídricas, que de acuerdo con Segura (2007), atribuye esta condición de pH ácido a aguas contaminadas con vertidos mineros o industriales. Pese a estas condiciones todos los puntos de muestreo presentaron unidades que están dentro del rango (4,5-9) establecidos por el Decreto 1594/84 para la conservación de la de flora y fauna, con pocas variaciones entre puntos y períodos de muestreo ( $p>0,05)$. A su vez la temperatura registró semejanzas entre puntos $25,4^{\circ} \mathrm{C}$ y $28,3^{\circ} \mathrm{C}$, correspondiente a aguas cálidas, típica de ambientes tropicales. Los grados más altos de temperatura pudieron obedecer a la incidencia de rayos solares por horas de muestreo; en el caso de esta variable no se presentaron diferencias estadísticamente significativas entre estaciones, 


\section{Características fisicoquímicas de afluentes del río Atrato. z Quesada Martínez}

Tabla 2. Resultados muestreo de variables fisicoquímicas

\begin{tabular}{|c|c|c|c|c|c|c|c|c|}
\hline \multirow[t]{2}{*}{ Estación } & \multirow[t]{2}{*}{$\begin{array}{l}\text { Fuente } \\
\text { hídrica }\end{array}$} & \multirow[t]{2}{*}{$\begin{array}{l}\text { Puntos de } \\
\text { muestreo }\end{array}$} & \multicolumn{2}{|c|}{$\begin{array}{c}\text { Oxígeno } \\
\text { disuelto }(\mathrm{mg} / \mathrm{l})\end{array}$} & \multicolumn{2}{|c|}{$\mathrm{pH}$} & \multicolumn{2}{|c|}{ Temperatura $\left({ }^{\circ} \mathrm{C}\right)$} \\
\hline & & & Muestreo 1 & Muestreo 2 & Muestreo 1 & Muestreo 2 & Muestreo 1 & Muestreo 2 \\
\hline \multirow[t]{3}{*}{1} & Quebrada & 1 & 3,9 & 4,3 & 6,9 & 6,71 & 26,9 & 25,4 \\
\hline & El Caraño & 2 & 3,1 & 4,74 & 6,7 & 6,97 & 27 & 25,6 \\
\hline & & 3 & 5,5 & 4,72 & 6,7 & 7 & 26,6 & 25,5 \\
\hline \multirow[t]{3}{*}{2} & Río Quito & 1 & 5,4 & 6,1 & 6,4 & 6,7 & 26,5 & 26,8 \\
\hline & & 2 & 5.2 & 6 & 6,1 & 6,4 & 26,7 & 26,8 \\
\hline & & 3 & 6,3 & 5,8 & 6,2 & 6,3 & 25,7 & 26,8 \\
\hline \multirow[t]{3}{*}{3} & Río Cabí & 1 & 5,5 & 6,69 & 6,2 & 6,74 & 25,6 & 27,2 \\
\hline & & 2 & 5,3 & 6,15 & 6,1 & 6,37 & 25,9 & 27,8 \\
\hline & & 3 & 5,5 & 6,35 & 6,1 & 6,7 & 26,4 & 28,3 \\
\hline \multirow[t]{3}{*}{4} & Quebrada & 1 & 2,7 & 3,36 & 6,96 & 6,97 & 27,2 & 26,1 \\
\hline & La Yesca & 2 & 2,7 & 3,62 & 7 & 6,97 & 27,5 & 26,5 \\
\hline & & 3 & 4,1 & 4 & 6,8 & 7,4 & 27,4 & 26,5 \\
\hline \multicolumn{3}{|c|}{ Kruskal-Wallis } & \multicolumn{2}{|c|}{16,8633} & \multicolumn{2}{|c|}{16,0717} & \multicolumn{2}{|c|}{2,536} \\
\hline & $\mathrm{p}$ & & \multicolumn{2}{|c|}{0,00075} & \multicolumn{2}{|c|}{0,001096} & \multicolumn{2}{|c|}{0,4688} \\
\hline
\end{tabular}

períodos y puntos de muestreo ( $\mathrm{p}>0,05)$ (Tabla 2$)$.

Las concentraciones de sólidos, como los sólidos suspendido (SS) mostraron una diferencia estadísticamente significativa $(\mathrm{p}<0,05)$ entre estaciones de muestreo; las concentraciones permanecieron casi constantes en las estaciones 1, 3 y 4, oscilando entre $44 \mathrm{mg} / \mathrm{l}$ y $98 \mathrm{mg} / \mathrm{l}$ en el primer muestreo y entre $19 \mathrm{mg} / \mathrm{l}$ y $79 \mathrm{mg} / \mathrm{l}$ en el segundo. La estación 2, presentó niveles más altos oscilando entre $359 \mathrm{mg} / \mathrm{l}$ y $369 \mathrm{mg} / \mathrm{l}$ en el primer muestreo y entre $448 \mathrm{mg} / 1$ y $456 \mathrm{mg} / 1$, estando por encima de límites establecidos por autores como Ramírez y Viña (1998) quienes han propuesto un límite de $150 \mathrm{mg} / \mathrm{l}$ de sólidos suspendidos en fuentes hídricas superficiales. Estos registros estuvieron directamente proporcionales a las concentraciones de turbidez que se presentaron entre 51 FAU y 365 FAU en el primer muestreo y entre 20 FAU y 468 FAU en el segundo. Los niveles elevados en las concentraciones de sólidos suspendidos y turbidez pueden estar relacionados con vertimientos directos de actividades mineras, que se desarrolla en los ríos Cabí y Quito, con mayor afluencia en este último, los cuales generan desprendimiento de grandes volúmenes de suelo, así como erosión de las rondas hídrica, que producen una alteración en los sedimentos en fuentes hídricas asociadas con la actividad. Al respecto IIAP (2014) reportó concentraciones de sólidos suspendidos desde 770 $\mathrm{mg} / \mathrm{l}$ en el río Quito y entre $38 \mathrm{mg} / \mathrm{l}$ y $753 \mathrm{mg} / \mathrm{l}$, sobre los 7 afluentes muestreados en este estudio; además se reportaron unidades de turbidez entre 123 FAU y 1100 FAU en la fuente hídrica principal del río Quito y entre 38 FAU y 877 FAU en sus afluentes. Las altas concentraciones de estas variables constituyen restricciones en el desarrollo de organismos acuáticos y procesos biológicos, porque los altos niveles de sólidos modifican la turbidez del agua incidiendo directamente sobre los procesos fotosintéticos, la producción primaria y las cadenas tróficas del ecosistema acuático (Seoánez 1999) (Tabla 3).

Los resultados de sólidos disueltos totales (TDS) con $\mathrm{p}<0,05$ de significancia estadística, oscilaron con entre $14,30 \mathrm{mg} / 1$ y $156,65 \mathrm{mg} / \mathrm{l}$ en el primer muestreo, y entre $6,68 \mathrm{mg} / \mathrm{l}$ y $28,70 \mathrm{mg} / 1$ 
Bioetnia Volumen 13, 2016

Tabla 3. Concentraciones de sólidos y variables asociadas

\begin{tabular}{|c|c|c|c|c|c|c|c|c|c|c|}
\hline \multirow[t]{2}{*}{ Estación } & \multirow[t]{2}{*}{$\begin{array}{l}\text { Fuente } \\
\text { hídrica }\end{array}$} & \multirow[t]{2}{*}{$\begin{array}{l}\text { Puntos de } \\
\text { muestreo }\end{array}$} & \multicolumn{2}{|c|}{$\begin{array}{l}\text { Sólidos } \\
\text { suspendidos (mg/l) }\end{array}$} & \multicolumn{2}{|c|}{ Turbidez (FAU) } & \multicolumn{2}{|c|}{$\begin{array}{l}\text { Sólidos disueltos } \\
\text { totales (mg/l) }\end{array}$} & \multicolumn{2}{|c|}{$\begin{array}{l}\text { Conductividad } \\
(\mu \mathrm{S} / \mathrm{cm})\end{array}$} \\
\hline & & & Muestreo 1 & Muestreo 2 & Muestreo 1 & Muestreo 2 & Muestreo 1 & Muestreo 2 & Muestreo 1 & Muestreo 2 \\
\hline \multirow[t]{3}{*}{1} & Quebrada & 1 & 60 & 65 & 65 & 68 & 58,5 & 21,77 & 93,6 & 46,8 \\
\hline & El Caraño & 2 & 73 & 51 & 73 & 75 & 66,6 & 18,17 & 105,8 & 39,3 \\
\hline & & 3 & 86 & 79 & 91 & 83 & 31,8 & 19,12 & 50,8 & 41,2 \\
\hline \multirow[t]{3}{*}{2} & Río Quito & 4 & 359 & 456 & 363 & 452 & 16,3 & 6,68 & 26,4 & 14,58 \\
\hline & & 5 & 365 & 448 & 365 & 438 & 14,3 & 6,96 & 28,2 & 15,51 \\
\hline & & 6 & 369 & 451 & 361 & 468 & 18,9 & 5,82 & 26,7 & 13,02 \\
\hline \multirow[t]{3}{*}{3} & Río Cabí & 7 & 82 & 33 & 88 & 35 & 29,9 & 7,93 & 46,7 & 17,8 \\
\hline & & 8 & 93 & 43 & 104 & 52 & 24,4 & 7,08 & 38,2 & 16,3 \\
\hline & & 9 & 98 & 19 & 106 & 20 & 19,9 & 7,81 & 26,6 & 17,93 \\
\hline \multirow[t]{3}{*}{4} & Quebrada & 10 & 44 & 45 & 51 & 46 & 80,6 & 28,7 & 129,3 & 62,5 \\
\hline & La Yesca & 11 & 70 & 51 & 73 & 52 & 156,65 & 31,1 & 252,3 & 68,2 \\
\hline & & 12 & 58 & 29 & 63 & 32 & 57,85 & 16,45 & 93,1 & 36,3 \\
\hline \multicolumn{2}{|c|}{ Kruskal-Wallis } & & \multicolumn{2}{|c|}{14,4713} & \multicolumn{2}{|c|}{15,2549} & \multicolumn{2}{|c|}{12,76} & \multicolumn{2}{|c|}{16,193} \\
\hline & $\mathrm{p}$ & & \multicolumn{2}{|c|}{0,002329} & \multicolumn{2}{|c|}{0,00161126} & \multicolumn{2}{|c|}{0,0051852} & \multicolumn{2}{|c|}{0,001} \\
\hline
\end{tabular}

en el segundo; en ambos períodos las concentraciones más altas de esta variable se presentaron en la estación 4. De igual forma se mostró una relación directa entre esta variable y la conductividad, la cual se registró entre $26,6 \mu \mathrm{s} / \mathrm{cm}$ y $252,3 \mu \mathrm{s} / \mathrm{cm}$ en el primer muestreo y entre 36,3 $\mu \mathrm{s} / \mathrm{cm}$ y $68,2 \mu \mathrm{s} / \mathrm{cm}$ presentándose en niveles altos en esta estación (quebrada La Yesca). Los datos registrados muestran niveles más altos en el primer muestreo con respecto al segundo para esta variable. Las variaciones presentadas en la conductividad pueden proporcionar información de la productividad de las fuentes monitoreadas al igual que procesos de descomposición de la materia orgánica. Autores como IDEAM(2010)y Ríos (2004), relacionan el incremento en los niveles de conductividad eléctrica en aguas superficiales con vertimientos domésticos de asentamientos humanos, situación que se presentó con los resultados de este muestreo, porque las estaciones 1 y 4 se encuentran presionadas por este tipo de vertimientos a lo largo de su cauce (Figura 3 ).

En relación con los nutrientes, el contenido de nitratos osciló entre $0 \mathrm{mg} / 1$ y $0,03 \mathrm{mg} / \mathrm{l}$ en el primer muestreo y entre 0 y $0,08 \mathrm{mg} / 1$ en el segundo, mostrando que no existe una diferencia significativa entre períodos y puntos de muestreo ( $\mathrm{p}>0,05)$; sin embargo, esta variable mostró diferencias estadísticamente significativas entre estaciones de muestreo $(p<0,05)$. Los nitritos mostraron un comportamiento diferente a los nitratos, se mantuvieron en concentraciones de $0,001 \mathrm{mg} / \mathrm{l}$ a $0,033 \mathrm{mg} / \mathrm{l}$ y entre $0,003 \mathrm{mg} / \mathrm{l}$ y 0,33 $\mathrm{mg} / \mathrm{l}$ en el primer y segundo muestreo respectivamente, evidenciándose diferencias estadísticas significativas entre muestreos $(p<0,05)$, pocas variaciones entre estaciones que no representaron diferencias estadísticas significativas $(\mathrm{p}>0,05)$. Los resultados muestran concentraciones bajas en relación con los nitritos y nitratos (Cooke et al. 2001); el Ministerio de Salud de Perú (2017) coincide en afirmar que las concentraciones de nitritos en aguas superficiales son bastante menores que $0.1 \mathrm{mg} / \mathrm{l}$. Los fosfatos por su parte se encontraron en mayores concentraciones en el segundo muestreo, oscilando entre $0,54 \mathrm{mg} / \mathrm{l} \mathrm{y}$ $2,5 \mathrm{mg} / 1$ y entre 0 y $2,5 \mathrm{mg} / 1$ en el primero. Esta variable no presentó diferencia estadística signi- 
Características fisicoquímicas de afluentes del río Atrato. z Quesada Martínez

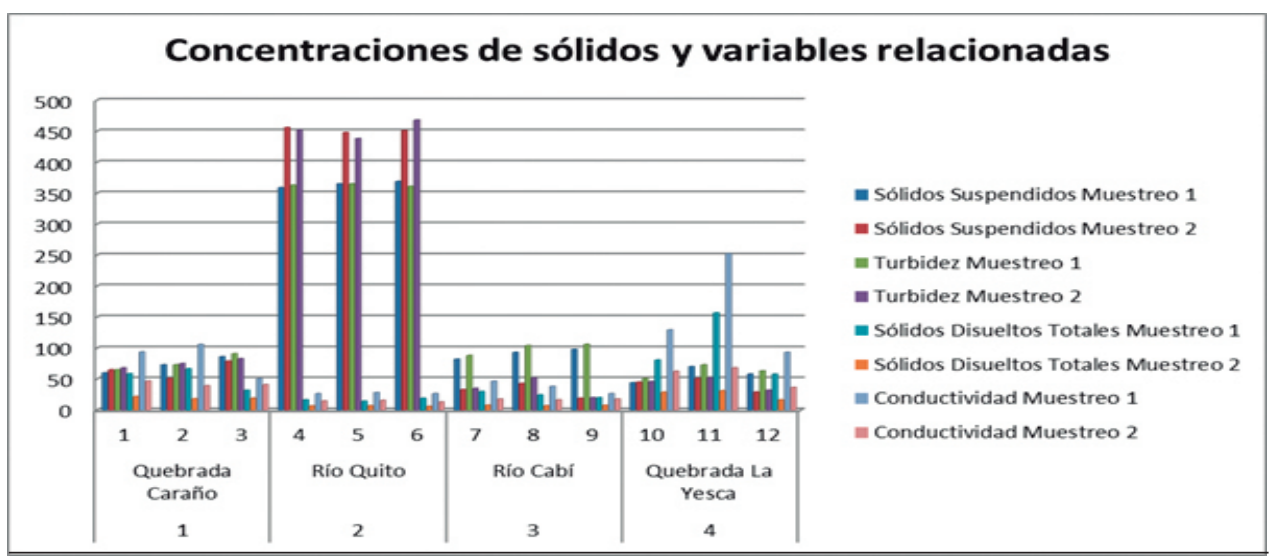

Figura 3. Variación de sólidos suspendidos, turbidez, sólidos disueltos totales y conductividad por estaciones y puntos de muestreo.

ficativa entre estaciones, períodos ni puntos de muestreo $(p>0,05)$. Esta variable registró altas concentraciones, de acuerdo con el Ministerio de Vivienda, Ordenamiento Territorial y Medio Ambiente de Uruguay (2007), quien establece un estándar de calidad para esta variable de 0,025 $\mathrm{mg} / \mathrm{l}$ para la conservación y establecimiento de organismos acuáticos. Los registros presentados están asociados con la materia orgánica presente en los residuos sólidos y líquidos de origen doméstico, así como a detergentes hechos con base en este compuesto, lo que puede generar el crecimiento acelerado de organismos vegetales como las algas (Tabla 4).

La DBO5 entendida como la cantidad de oxígeno requerido por los microorganismos para degradar la materia orgánica se mantuvo semejante con respecto a los puntos de muestreo, presentando un incremento significativo en el punto 2 de la estación 4 en ambos períodos de

Tabla 4. Concentraciones de nutrientes por estación y puntos de muestreo

\begin{tabular}{|c|c|c|c|c|c|c|c|c|}
\hline \multirow[t]{2}{*}{ Estación } & \multirow[t]{2}{*}{ Fuente hídrica } & \multirow{2}{*}{$\begin{array}{l}\text { Puntos de } \\
\text { muestreo }\end{array}$} & \multicolumn{2}{|c|}{ Nitratos (mg/l) } & \multicolumn{2}{|c|}{ Nitritos (mg/l) } & \multicolumn{2}{|c|}{ Fosfatos (mg/l) } \\
\hline & & & Muestreo 1 & Muestreo 2 & Muestreo 1 & Muestreo 2 & Muestreo 1 & Muestreo 2 \\
\hline \multirow[t]{3}{*}{1} & Quebrada & 1 & 0,03 & 0,08 & 0,026 & 0,021 & 2,5 & 1,32 \\
\hline & El Caraño & 2 & 0,03 & 0,03 & 0,033 & 0,015 & 2,5 & 1,67 \\
\hline & & 3 & 0,03 & 0,03 & 0,002 & 0,023 & 2,63 & 2,5 \\
\hline \multirow[t]{3}{*}{2} & Río Quito & 4 & 0,03 & 0,03 & 0,011 & 0,002 & 2,5 & 2,5 \\
\hline & & 5 & 0,03 & 0,03 & 0,001 & 0,016 & 0.27 & 1,46 \\
\hline & & 6 & 0,03 & 0,03 & 0,001 & 0,026 & 0 & 0,18 \\
\hline \multirow[t]{3}{*}{3} & Río Cabí & 7 & 0,03 & 0 & 0,007 & 0,026 & 1,24 & 1,82 \\
\hline & & 8 & 0,03 & 0 & 0,003 & 0,063 & 2,5 & 1,21 \\
\hline & & 9 & 0,03 & 0,03 & 0,002 & 0,014 & 0,93 & 1,71 \\
\hline \multirow[t]{5}{*}{4} & Quebrada & 10 & 0 & 0 & 0,029 & 0,029 & 2,5 & 1,46 \\
\hline & La Yesca & 11 & 0,03 & 0 & 0,022 & 0,027 & 2,5 & 1,11 \\
\hline & & 12 & 0,03 & 0 & 0,003 & 0,33 & 0,72 & 0,54 \\
\hline & Kruskal-Wallis & & \multicolumn{2}{|c|}{9,66138} & \multicolumn{2}{|c|}{6,23482} & \multicolumn{2}{|c|}{4,69691} \\
\hline & $p$ & & \multicolumn{2}{|c|}{0,0216744} & \multicolumn{2}{|c|}{0,100726} & \multicolumn{2}{|c|}{0,195381} \\
\hline
\end{tabular}




\section{Bioetnia Volumen 13, 2016}

muestreo ( $p>0,05)$, correspondiendo a aguas con bajo contenido de materia orgánica y suficientes niveles de oxígeno disuelto para oxidarla y al mismo tiempo reoxigenarse. Con respecto a las grasas y aceites, existe una diferencia estadísticamente significativa entre la media estaciones con respecto a esta variable $(\mathrm{p}<0,05)$, registrándose concentraciones entre $4 \mathrm{mg} / \mathrm{l}$ y $10,7 \mathrm{mg} /$ para el segundo muestreo y entre $4 \mathrm{mg} / \mathrm{y} 8,8 \mathrm{mg} /$ en el segundo muestreo. En relación con las estaciones de muestreo, no se presentaron diferencias estadísticamente significativas $(p>0,05)$ entre estaciones, períodos, ni puntos de muestreo, los registros mostraron que la estación 4 presentó las mayores concentraciones de esta variable, que de acuerdo con el Decreto 1594/84 no debe superar $0,01 \mathrm{mg} / \mathrm{l}$ para la conservación de organismos acuáticos. Estas concentraciones estuvieron asociadas con el vertimiento de sustancias aceitosas de origen doméstico como restos de alimentos y aguas residuales, al igual que sustancias industriales como lubricantes, combustibles y aceites industriales asociados con procesos mineros en la zona (Tabla 5).
La calidad fisicoquímica del agua en las estaciones monitoreadas está directamente asociada con el tipo de vertimientos directos que reciben en su cauce, lo que a su vez puede generar un deterioro en la calidad del agua del río Atrato como fuente receptora. El análisis de correspondencia sin tendencia (DCA) agrupa las variables nitratos, sólidos suspendidos y turbidez en un componente que explica $91,2 \%$ de la variabilidad observada en las estaciones de muestreo y a este se asocian todos los muestreos realizados en todas las estaciones, indicando que son las variables que ejercen mayor presión sobre la calidad del agua (Figura 4). El DCA muestra además que la turbidez y los sólidos suspendidos presentan mayor concentraciones y están asociadas con niveles más altos de represamiento y vertimiento, que para el caso en particular, se asocia con el desarrollo de actividades mineras que ejercen presión sobre las cuencas del río Quito y Cabí, ocasionando impactos significativos sobre los ecosistemas hídricos asociados con la afectación de las condiciones fisicoquímicas, alteración del cauce y erosión de las rondas hídricas (IIAP

Tabla 5. Concentraciones de DBO5, grasas y aceites

\begin{tabular}{|c|c|c|c|c|c|c|}
\hline \multirow[t]{2}{*}{ Estación } & \multirow[t]{2}{*}{ Fuente hídrica } & \multirow{2}{*}{$\begin{array}{l}\text { Puntos de } \\
\text { muestreo }\end{array}$} & \multicolumn{2}{|c|}{ BDO5 (mg/l) } & \multicolumn{2}{|c|}{ Grasas y aceites (mg/l) } \\
\hline & & & Muestreo 1 & Muestreo 2 & Muestreo 1 & Muestreo 2 \\
\hline \multirow[t]{3}{*}{1} & Quebrada El Caraño & 1 & 2 & 2 & 4 & 5 \\
\hline & & 2 & 2 & 2 & 4,4 & 4,4 \\
\hline & & 3 & 2 & 2 & 4 & 6,9 \\
\hline \multirow[t]{3}{*}{2} & Río Quito & 4 & 2 & 2 & 4 & 4 \\
\hline & & 5 & 2 & 2 & 4 & 4,2 \\
\hline & & 6 & 2 & 2 & 4 & 4 \\
\hline \multirow[t]{3}{*}{3} & Río Cabí & 7 & 2 & 2 & 4 & 7,8 \\
\hline & & 8 & 2 & 2 & 4 & 7 \\
\hline & & 9 & 2 & 2 & 4 & 5,6 \\
\hline \multirow[t]{3}{*}{4} & Quebrada La Yesca & 10 & 2 & 2 & 4 & 4,1 \\
\hline & & 11 & 5,27 & 4,26 & 10,7 & 8,8 \\
\hline & & 12 & 2 & 2 & 4 & 8,1 \\
\hline & Kruskal-Wallis & & \multicolumn{2}{|c|}{6,26087} & \multicolumn{2}{|c|}{4,75882} \\
\hline & $\mathrm{p}$ & & \multicolumn{2}{|c|}{0,0995834} & \multicolumn{2}{|c|}{0,190331} \\
\hline
\end{tabular}




\section{Características fisicoquímicas de afluentes del río Atrato. z Quesada Martínez}

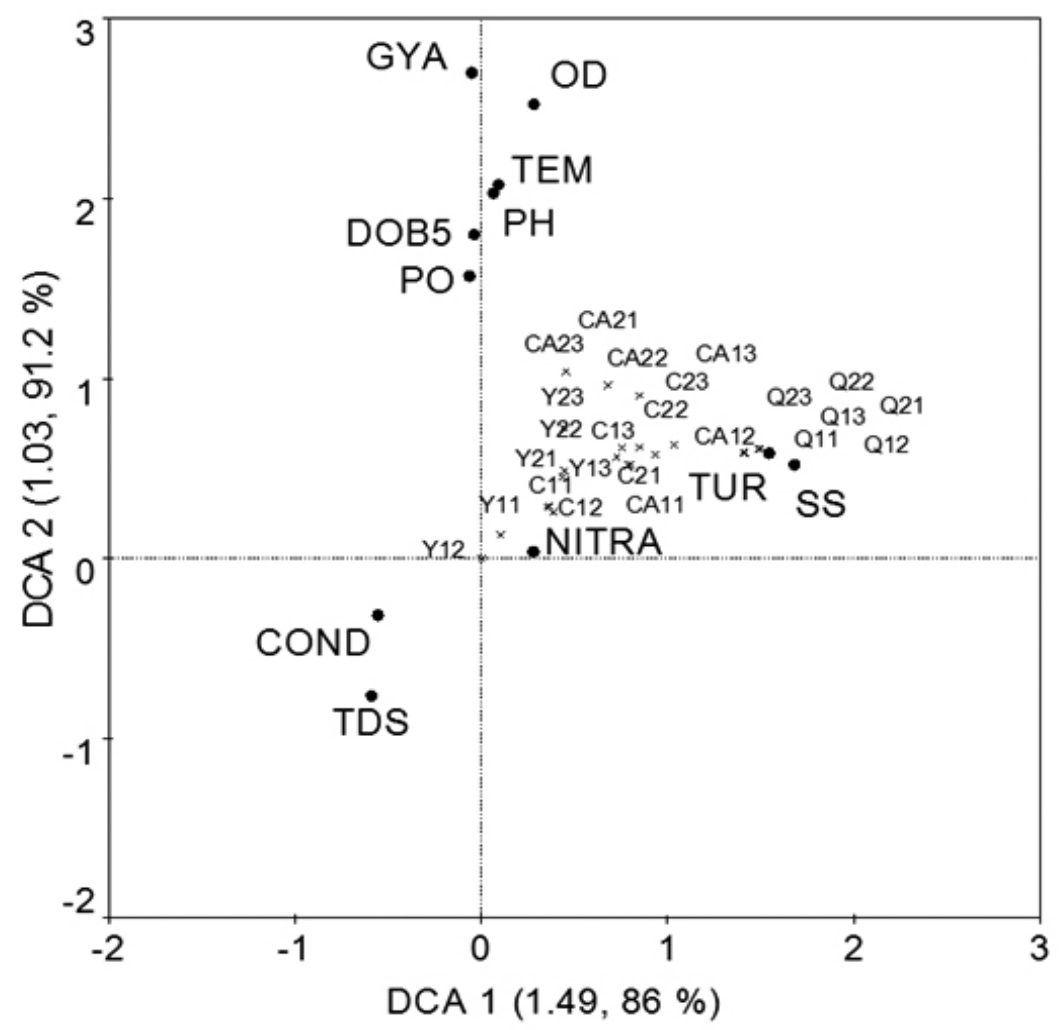

Figura 4. Análisis de correspondencia sin tendencia (DCA). Estaciones ríos Quito, Cabí y las quebradas La Yesca y EI Caraño.

2014). Por su parte, las variables asociadas con la actividad doméstica son los nutrientes, que como se muestra en la Figura 4, están asociados con las estaciones de muestreo ubicadas en las quebradas La Yesca y El Caraño, y el río Cabí, pese a que se encontraron en concentraciones mínimas, estas estuvieron directamente relacionadas con vertimientos de origen doméstico, lo que coincide con afirmaciones hechas por la OMS (2004), quienes expresan que la presencia de nitratos se puede deber a la aplicación excesiva de fertilizantes o a la filtración de aguas residuales u otros residuos orgánicos a las aguas superficiales.

De acuerdo con el DCA, las variables como oxígeno disuelto, el $\mathrm{pH}$, la temperatura, y la DBO5, no mostraron correspondencia significativa entre estaciones de muestreo, lo que puede estar relacionado con período de recolección de los datos, cambios climáticos y el caudal, porque estas variables están más relacionadas con estas condiciones del ambiente, por lo tanto, no ejercieron cambios significativos en las condiciones fisicoquímicas del agua. Tanto la temperatura como el oxígeno disuelto están sometidos a fluctuaciones de las corrientes de agua, lo que se presenta como una situación normal debida, entre otras razones, a las variaciones del clima y a las transiciones altitudinales. De manera particular, la quebrada La Yesca presentó niveles de oxigenación bajos, asociados con altas concentraciones de materia orgánica de origen doméstico que son descompuestos por bacterias aeróbicas que consecuentemente disminuyen el oxígeno. Se presentaron otros niveles de agrupación, relacionados con las variables conductividad y sólidos disueltos totales, las cuales están relacionados 


\section{Bioetnia Volumen 13, 2016}

con el contenido de sales minerales en el agua. Pese a que la conductividad está relacionada con zonas de contaminación alrededor de un afluente, sus concentraciones mínimas evidenciaron poca influencia sobre las condiciones fisicoquímicas del agua en las estaciones de muestreo. En términos generales, pese a que variables como grasas y aceites, fosfatos, DBO5, y la temperatura, no presentaron importancia estadística significativa, estas inciden en el estado de la calidad fisicoquímica de las fuentes hídricas muestreadas, porque se presentaron concentraciones elevadas de grasas y aceites, así como de nutrientes del fosfato que alteran el equilibrio ecológico del agua.

\section{Conclusiones}

De manera general las variables fisicoquímicas evaluadas son una expresión de un conjunto de factores que influyen en las dinámicas de las fuentes hídricas monitoreadas y están asociadas con el tipo de actividades que se realizan a lo largo de sus cauces, al igual que factores ambientales y climáticos que están inherentemente relacionados con los cambios en la calidad de ecosistemas acuáticos. El análisis de Kruskal-Wallis mostró variables estadísticamente significativas como el oxígeno disuelto, sólidos suspendidos, conductividad, turbidez, sólidos disueltos totales y los nitratos, los cuales estuvieron asociados con dos fuentes de contaminación como los vertimientos domésticos y los de origen industrial (actividad minera), situación que se corroboró con los dos niveles de asociación que resultaron del análisis de correspondencia sin tendencia (DCA).

Las estaciones asociadas con contaminación de origen doméstico, caracterizadas por contener sustancias biodegradables y compuestos químicos domésticos como detergentes, compuestos de cloro y microorganismos patógenos fueron las quebradas La Yesca, El Caraño y el río Cabí, y las estaciones asociada con contaminación de origen industrial sobre todo de vertimientos mineros fue el río Quito. El DCA agrupó los puntos de muestreo y las variables fisicoquímicas asociadas con este tipo de contaminación, como son los sólidos suspendidos, la turbidez y los nitratos. Con los resultados obtenidos se recomienda la implementación de sistemas de saneamiento que reduzcan los niveles de contaminación, al igual que el seguimiento, monitoreo e implementación de sistemas de reducción de agentes contaminantes por actividad minera. La implementación de medidas de control permitirá el uso y conservación de los sistemas hídricos monitoreados, así como el conocimiento de los aportes contaminantes urbanos que ingresan al río Atrato por estas fuentes hídricas afluentes en el municipio de Quibdó.

\section{Literatura citada}

Corporación Autónoma Regional para el Desarrollo Sostenible del Chocó (CODECHOCO). 2009. POMCA, Plan de manejo y ordenamiento de la cuenca del río Cabí. Quibdó: CODECHOCÓ.

Cooke R, Griggs J, Sánchez L, Díaz C, Carvajal D. 2001. Recopilación y presentación de datos de recursos ambientales y culturales en la región occidental de la cuenca del canal de Panamá. Volumen 4. Calidad Ambiental. Informe Final de la Región Occidental de la Cuenca del Canal, Consorcio TLBG UP STRI.

Empresas Públicas de Quibdó en liquidación, EPQ. 2017. Diagnóstico de la prestación de los servicios públicos de acueducto, alcantarillado y aseo en el municipio de Quibdó. Informe técnico. URL disponible en: https:// www.quibdo-choco.gov.co/Institucional/Banco $\% 20$ de\%20Documentos/Medio\%20Ambiente/Diagn\%C3\%B3stico\%20prestaci\%C3\%B3n\%20de \%20 los\%20\%20servicios\%20publicos\%20Quibdo. $\% 20$ COMPLETO.pdf

Faustino J. 1986. Criterios para la clasificación de los problemas y soluciones en la conservación de suelos $y$ aguas. Turrialba: Centro Agronómico Tropical de Investigación y Enseñanza (CATIE); 60 pp.

Hinrichsen D, Robey B, Upadhyay UD. 1998. Soluciones para un mundo con escasez de agua. Baltimore: Population Reports. Serie M, No 14, Johns Hopkins School of Public Health, Population Information Program; $147 \mathrm{pp}$.

IDEAM. 2010. Calidad del agua superficial en Colombia. Estudio Nacional del Agua. Informe técnico. Bogotá: IDEAM; 52 pp. URL disponible en: http:// 


\section{Características fisicoquímicas de afluentes del río Atrato. z Quesada Martínez}

oab.ambientebogota.gov.co/es/con-la-comunidad/ES/ estudio-nacional-del-agua-2010

IDEAM. 2013. Lineamientos conceptuales ymetodológicos para la evaluación regional del agua. Bogotá: IDEAM; 218 pp. URL disponible en: http://documentacion. ideam.gov.co/openbiblio/bvirtual/022653/022653.htm

Instituto de Investigaciones Ambientales del Pacífico(IIAP). 2014. Evaluación de la calidad fisicoquímica y ecológica del río Quito como herramienta de análisis de los impactos ocasionados por la minería y su importancia ecosistémica y sociocultural. Informe técnico. Quibdó: IIAP; $124 \mathrm{pp}$.

Instituto de Investigaciones Ambientales del Pacífico(IIAP). 2012. Caracterización integral de la microcuenca El Caraño del municipio de Quibdó con fines de ordenamiento y manejo. Documento de investigación. Quibdó: IIAP; 119 pp.

Ministerio de Agricultura. Decreto 1594 de 1984. Usos del agua y residuos líquidos. Bogotá: MinAgricultura. URL disponible en: http://www.alcaldiabogota.gov.co/ sisjur/normas/Norma1.jsp? $\mathrm{i}=18617$

Ministerio de Salud, República de Perú. 2017. Parámetros organolépticos. Gestión de agua. Grupo de Estudio Técnico Ambiental; 145 pp. URL disponible en: http:// www.digesa.minsa.gob.pe/DEPA/informes tecnicos/ GRUPO\%20DE\%20USO\%201.pdf

Ministerio de Vivienda, Ordenamiento Territorial y Medio Ambiente de Uruguay. 2007. La calidad del agua del río Uruguay, resultados de las tres primeras campañas. Montevideo: Dirección Nacional de Medio Ambiente. Organización Mundial de la Salud. 2004. Guías para la calidad del agua potable. $3^{\mathrm{a}}$ ed. Volumen 1. Ginebra: OMS; 408 pp. URL disponible en: http://www.who.int/ water sanitation health/dwq/gdwq3 es fulll lowsres. pdf

RamírezA, Viña G. 1998. Limnología colombiana: aportes a su conocimiento y estadísticas de análisis. Bogotá: Fundación Universidad Jorge Tadeo Lozano; 293 pp.

Ríos SP. 2004. Caracterización fisicoquímica y microbiológica de la quebrada Cuchicute ubicadas en los municipios de San Gily Curití. Bucaramanga: Escuela de Química, Facultad de Ciencias, Universidad de Santander.

Rojas MG, Calderón C, Ríos C. 2007. Determinación de las características socioambientales y de infraestructura de la microcuenca La Yesca en el municipio de Quibdó, departamento del Chocó. Quibdó: Universidad Tecnológica del Chocó; 31 pp.

Segura LE. 2007. Estudio de antecedentes sobre la contaminación hídrica en Colombia. Bogotá: Escuela Superior de Administración Pública (ESAP): 142 pp. URL disponible en: http://cdim.esap.edu.co/BancoMedios/ Documentos $\% 20 \mathrm{PDF} /$ estudio $\% 20 \mathrm{de} \% 20$ antecedentes $\% 20$ sobre $\% 201 \mathrm{a} \% 20$ contaminaci $\% \mathrm{C} 3 \% \mathrm{~B} 3 \mathrm{n} \% 20$ h\%C3\%ADdrica.pdf

Seoánez M. 1999. Ingeniería del medioambiente aplicada al medio natural continental. $2^{\mathrm{a}}$ ed. Madrid: Ediciones Mundi-Prensa Libros; 701 pp.

Wall JRD eds. 1981. A management plan for the Acelhuate River catchment, El Salvador: soil conservation, river stabilization and water pollution control. London: Land Resources Development Centre; 158 pp. 